Faculty \& Staff Scholarship

2009

Solution Techniques for a Crane Sequencing Problem

Jin Shang

Alan McKendall

Follow this and additional works at: https://researchrepository.wvu.edu/faculty_publications

Part of the Operations Research, Systems Engineering and Industrial Engineering Commons 


\title{
Solution techniques for a crane sequencing problem
}

\section{Jin Shang}

Quality Planning Corporation, 388 Market Street, Suite 750,

San Francisco, CA 94111, USA

Fax: +415 2688648

E-mail: jshang@qualityplanning.com

\author{
Alan R. McKendall* \\ Department of Industrial and \\ Management Systems Engineering, \\ West Virginia University, \\ 325A Mineral Resources Building, \\ P.O. Box 6070, \\ Morgantown, WV 26506, USA \\ Fax: +3042934970 \\ E-mail: armckendall@mail.wvu.edu \\ *Corresponding author
}

\begin{abstract}
In the areas of power plant maintenance, shipyard and warehouse management, resources (items) assigned to locations need to be relocated. It is essential to develop efficient techniques for relocating items to new locations using a crane such that the sum of the cost of moving the items and the cost of loading/unloading the items is minimised. This problem is defined as the crane sequencing problem (CSP). Since the CSP determines the routes for a crane to relocate items, it is closely related to some variants of the travelling salesman problem. However, the CSP considers the capacities of locations and intermediate drops (i.e. preemptions) during a multiple period planning horizon. In this article, a mathematical model and hybrid ant systems are developed for the CSP. Computational experiments were conducted to evaluate the performances of the proposed techniques, and results show that the proposed heuristics are effective.
\end{abstract}

Keywords: crane sequencing problem; hybrid ant systems; integer programming; meta-heuristics; simulated annealing.

Reference to this paper should be made as follows: Shang, J. and McKendall, A.R. (xxxx) 'Solution techniques for a crane sequencing problem', Int. J. Mathematics in Operational Research, Vol. x, No. x, pp.xx-xx.

Biographical notes: Jin Shang is a Senior Software Engineer at Quality Planning Corporation in San Francisco, CA. He earned his PhD and MS degree in Industrial Engineering from West Virginia University. Currently, he conducts research on the use of algorithms in database keyword search and algorithm implementation. He also has experience in using different heuristics to solve real industrial problems such as the dynamic facility layout and crane sequencing problems. His research interests are developing heuristics for various combinatorial problems. 
Alan R. McKendall is an Associate Professor in the Department of Industrial and Management Systems Engineering at West Virginia University. He received his $\mathrm{PhD}$ and $\mathrm{MS}$ in Industrial Engineering and MS in Applied Mathematics from the University of Missouri, Columbia. His main research interest is in developing efficient algorithms in the areas of logistics and scheduling. He has published in journals such as Computers and Operations Research, Information Sciences, Int. J. Operational Research and Int. J. Production Research. Currently, he is on the Editorial Board of the Open Operational Research Journal and Int. J. Mathematics in Operational Research.

\section{Introduction}

The crane sequencing problem (CSP) is the task of determining the sequences in which a single overhead crane moves items (or resources) to their new locations with respect to minimising the total travel and loading/unloading costs. More specifically, when items are rearranged (reassigned to new locations), the crane is used to relocate the items. First, the crane arrives at the location of an item to be moved. Second, the item is loaded onto the crane. Third, the item is moved from its original position to its new position if the position is available. Otherwise, the item is moved to a temporary location. Nevertheless, the item is unloaded from the crane. This process is repeated for each item being rearranged. An item assigned to a temporary location is moved to its assigned location when the location is available and either the crane is at its temporary location or at the end of the sequence of moves. Hence, the CSP determines the order in which the items should be moved such that the total cost is minimised. The total cost consists of two major components. First, loading/unloading cost is the cost of loading the items onto the crane plus the cost of unloading the items. Second, the crane travel cost is the cost of moving the items from their original locations to their destination locations using the crane, which is the sum of the product of the distances the crane travels and the cost of moving items per distance unit.

The assumptions for the CSP are as follows.

1 The location of each item in each period, the distances between locations, the capacities of the locations, initial location of the crane, cost of moving items per distance unit and the costs of loading/unloading items are known.

2 All items may not be relocated at each period.

3 The capacity for a single overhead crane is one unit of an item.

4 After the second period, the initial position of the crane is the last position of the crane in the previous period.

5 Preemption can occur, and any available locations can be used as temporary storage locations.

6 The resources are fixed at their locations if they are not scheduled to be moved at a specific time period. 
7 The objective of the CSP is to find the sequences in which items are to be moved by the crane such that the sum of the loading/unloading and crane travel costs is minimised.

The CSP is a problem which considers multiple time periods (i.e. items may be reassigned to locations in different periods). As a result, the CSP determines the crane route for each period items are rearranged. It is important to note that all items may not be rearranged from one period to the next as stated in Assumption (2). Without loss of generality, the initial location of the crane at the beginning of each period, not including the initial period, is the last position of the crane in the previous period as defined in Assumption (4). However, if the initial position of the crane at the beginning of each period is known, the problem can be solved as a series of static CSPs, which is much easier than the CSP defined in this article. Furthermore, it is easy to modify the mathematical model and heuristics below to consider this case. In Assumption (5), temporary storage locations can be used to store items temporarily when:

1 The destination location of an item currently being moved is at full capacity. In this case, either the item has to be moved to another available location (called a temporary storage space) or the item is moved at a later time when the destination location is available.

2 Routes of the crane can be improved (i.e. the sum of total travel and loading/unloading costs for the crane is reduced) if temporary storage space is used.

Both of these cases are illustrated below. Nevertheless, once an item is moved to a temporary storage space, it is stored there until its assigned location is available and either the crane is at its temporary location or after all the other items has been moved to their assigned locations as stated previously. Also, available locations (temporary locations) are defined as storage locations not at full capacities and workspaces that are currently available (i.e. no activities are currently being performed in the workspaces). In the literature (e.g. Charikar, Khuller and Raghavachari, 2002), when an item can be put into an intermediate location before it is delivered to its final destination location, it is called 'preemption' or 'intermediate drop'. There are different variations of the CSP where locations can be workspaces (i.e. locations use to perform activities which require resources) and/or storage locations (locations used to store resources not to perform activities). For instance, during planned outages at electric power plants, the locations can be defined as workspaces where outage activities are performed and resources (items) are required to perform the activities. Also, within the same application, locations can be either workspaces, as defined above, or storage spaces where idle resources are stored until they are needed to perform an activity. On the other hand, locations can be defined as storage locations only where products are stored until they are needed (e.g. as in storing products in a warehouse). Nevertheless, the techniques presented in this article can be applied to all the above scenarios.

The CSP was first defined by McKendall et al. (2006) in which items, during outages at electric power plants, are to be moved using a single overhead crane such that the total distance travelled by the crane is minimised. The authors presented a simulated annealing heuristic for the CSP. McKendall and Shang (2008) presented several tabu search heuristics for the CSP, as defined in this article (i.e. items are to be moved using a single overhead crane such that the sum of the crane travel cost and loading/unloading costs is minimised). The first tabu search heuristic is a simple tabu search heuristic, the second is 
a probabilistic tabu search heuristic, and the third adds diversification and intensification strategies to the first. These are the only papers, known to the authors, which consider the CSP defined in this article.

Besides determining the order in which items are relocated during outages at electric power plants, the CSP has many potential applications in the real world. Another application of the CSP occurs in the context of warehouse rearrangement. Since the demand of products is dynamic and some products may become obsolete, warehouse managers may rearrange the layout of their products in warehouses. For example, products with high demand usually are located close to the input/output locations. As a result, the locations of products may change. Therefore, the CSP can be used to determine the sequence in which the crane (or vehicle) moves the products reassigned to new locations in a warehouse. This problem is called the warehouse rearrangement problem and was presented in Christofides and Colloff (1973). Also, the CSP can be used to determine the order in which a crane removes containers from ships or vice versa (i.e. determine the order in which a crane loads containers onto ships).

In this article, a binary integer linear program for the CSP is developed to obtain optimal solutions for small-size problems. Since only small-size problems can be solved optimally in reasonable computational time, hybrid ant systems (HASs) are developed to solve large-size CSPs. The article is organised as follows. Section 2 presents a mathematical model for the CSP. Also, a small CSP instance is presented. Section 3 describes the proposed HASs to solve the CSP, and the computational results are summarised in Section 4. Section 5 concludes the article.

\section{Mathematical formulation}

In this section, a binary integer linear program is presented for the CSP. First the notation is given below. The indices are as follows:

$i, j, h=1, \ldots, L$, where $L$ is the number of locations

$r=1, \ldots, R+1$, where $R$ is the number of items (or resources) and $R+1$ represents a dummy item (empty move)

$k, k_{2}=1, \ldots, K$, where $K$ is an upper bound on the number of moves

$t=1, \ldots, T$, where $T$ is the total number of periods.

The parameters are given below:

$A_{t}=$ set of items to be moved at period $t$ (not including $\left.R+1\right)$

$B_{t}=A_{t} \cup\{R+1\}$, which is a set of items to be moved at period $t$ including $R+1$

$Q_{t}=$ set of locations which cannot be used as temporary storage locations because activities are being performed in those locations at period $t$

$U B_{t}=$ upper bound for the number of moves in period $t$, where $U B_{t}=3\left|A_{t}\right|$ and

$K=\max \left\{U B_{t}\right\}$

$d_{i j}=$ distance between locations $i$ and $j$ 
$l_{r t}=$ the location of item $r$ at period $t$

$n_{j t}=$ the number of items in location $j$ at period $t$

$p=$ the initial location of the crane at the beginning of period 2 (assume location 1)

$C_{j}=$ capacity of location $j$

$M=$ a larger number

$W_{1}=$ travel cost per distance unit

$W_{2}=$ loading/unloading cost per item moved.

The decision variables are defined as

$$
x_{t, k, r, i, j}= \begin{cases}1, & \text { If at the end of period } t, \text { item } r \text { is moved from location } i \text { to } j \text { at } k \text { th crane moved; } \\ 0, & \text { Otherwise. }\end{cases}
$$

The binary integer linear program for the CSP is as follows.

$$
\begin{aligned}
\text { Minimise total cost }= & W_{1} \sum_{t} \sum_{k \leq U B_{t}} \sum_{r \in B_{t}} \sum_{i} \sum_{j} d_{i j} x_{t, k, r, i, j} \\
& +W_{2} \sum_{t} \sum_{k \leq U B_{t}} \sum_{r \in A_{t}} \sum_{i} \sum_{j} x_{t, k, r, i, j}
\end{aligned}
$$

subject to:

$$
\begin{aligned}
& \sum_{k<=k 2} \sum_{i} \sum_{j} x_{t, k, r, i, j} \leq M \sum_{k<=k 2} \sum_{j} x_{t, k, r, i=l_{r t}, j} \quad \forall t, k 2, r \in A_{t} \\
& \sum_{k} \sum_{j} x_{t, k, r, i=l_{r t}, j}=1 \quad \forall t, r \in A_{t} \\
& M\left(1-\sum_{k<=k 2} \sum_{i} x_{t, k, r, i, j=l_{r t+1}}\right) \geq \sum_{k>=k 2+1} \sum_{i} \sum_{j} x_{t, k, r, i, j} \quad \forall t, k 2, r \in A_{t} \\
& \sum_{k} \sum_{i} x_{t, k, r, i, j=l_{r t+1}}=1 \quad \forall t, r \in A_{t} \\
& n_{j t}+\sum_{k<=k 2} \sum_{r \in A_{t}} \sum_{i} x_{t, k, t, i, j}-\sum_{k<=k 2-1} \sum_{r \in A_{t}} \sum_{i} x_{t, k, t, j, i} \leq C_{j} \quad \forall t, k 2, j \\
& \sum_{r \in B_{t}} \sum_{i} \sum_{j} x_{t, k, r, i, j} \leq 1 \quad \forall t, k \\
& \sum_{r \in B_{t}} \sum_{j} x_{t=1, k=1, r, i=p, j}=1 \\
& \sum_{r \in B_{t}} \sum_{i} x_{t, k, r, i, j=h} \leq \sum_{r \in B_{t}} \sum_{j} x_{t, k+1, r, i=h, j}+1-\sum_{r \in B_{t}} \sum_{i} \sum_{j} x_{t, k+1, r, i, j} \quad \forall t, h, k<K
\end{aligned}
$$




$$
\begin{aligned}
& \sum_{r \in B_{t}} \sum_{i} x_{t-1, k=k 2, r, i, j=h}-\sum_{r \in B_{t}} \sum_{i} \sum_{j} x_{t-1, k=k 2+1, r, i, j} \\
& \quad \leq \sum_{r \in B_{t}} \sum_{j} x_{t, k=1, r, i=h, j} \quad \forall t>2, h, k 2 \\
& \sum_{i} x_{t, k=k 2, r, i, j=h} \leq \sum_{k>k 2} \sum_{j} x_{t, k, r, i=h, j} \quad \forall t, r \in A_{t}, h \neq l_{r t+1}, k 2 \\
& \sum_{i} \sum_{k<k 2} x_{t, k, r, i, j=h} \geq \sum_{j} x_{t, k=k 2, r, i=h, j} \quad \forall t, r \in A_{t}, h \neq l_{r t}, k 2 \\
& \sum_{r} \sum_{i} \sum_{j} x_{t, k, r, i, j} \geq \sum_{r} \sum_{i} \sum_{j} x_{t, k=k+1, r, i, j} \quad \forall t, k<K \\
& \sum_{k} \sum_{r \in A_{t}} \sum_{i} x_{t, k, r, i, j=Q_{t}}=0 \quad \forall t, Q_{t} \\
& x_{t, k, r, i, j}=0 \text { or } 1 \quad \forall t, k, r, i, j .
\end{aligned}
$$

The objective function (0) minimises the sum of the total travel and loading/unloading costs, where the first term considers total crane travel cost and the second term considers total loading/unloading cost. Constraint (1) ensures that no item (or resource) can be moved before it is moved from its initial position, and constraint (2) ensures that each item being rearranged is always moved from its initial location. Similarly, constraint (3) ensures that no item can be moved after it is moved to its destination location, and constraint (4) guarantees that each item being rearranged is always moved to its destination location. Constraint (5) restricts an item from being moved to a location at full capacity. Constraint (6) ensures that for each possible crane move, the crane can move only between two locations and can carry at most one item. Constraint (7) makes sure that the crane starts from its initial location $p$ at the initial period. Constraint (8) guarantees that each crane move destination is the starting position of the next crane move. For instance, if a crane moves from locations 1 to 5 , then the starting position of the next crane move is location 5. Constraint (9) ensures that the last crane move destination in period $t-1$ is the initial position of the first crane move in period $t$. For example, if the last move in period 3 terminates at location 4 , then the first move in period 4 starts at location 4 . Constraints (10) and (11) are used to temporarily store items in temporary storage locations. Constraint (10) ensures that an item not at its destination location (i.e. either at its initial location or a temporary location) at the current move will be moved to its destination location later. Constraint (11) allows an item to be moved to a temporary storage space. Constraint (12) ensures that once a crane starts to rearrange items in a period, it does not stop until all items have been rearranged. Constraint (13) restricts workspaces, currently used to perform activities, from being used as temporary storage spaces. Last, the restrictions on the decision variables are given in constraint (14).

It is important to note that the condition which requires an item stored in a temporary storage space to be moved to its assigned location when the location is available and either the crane is at its temporary location or after all the other items has been moved to their assigned location is relaxed for the mathematical model. In other words, an item stored in a temporary storage space is moved to its assigned location when the location is 
available and either the crane is at or close to its temporary location or after all the other items has been moved to their assigned locations. This increases the solution space tremendously. Since the CSP is computationally intractable, only small-size CSPs can be solved in reasonable time using the mathematical model. Therefore, in Section 3, HASs are presented for the CSP, but first the solution representation for the HASs is defined, followed by a presentation of a small CSP instance.

In this article, $\pi_{t}$ is used to represent an ordered list of items (i.e. a sequence of items) to be moved by the crane at the beginning of period $t$ such that $t=2, \ldots, T$. In other words, $\pi_{t}$ is a permutation of items needed to be moved at the beginning of period $t$ and is represented as follows:

$$
\pi_{t}=\left(\pi_{1 t}, \pi_{2 t}, \ldots, \pi_{n_{t}}\right), \text { for } t=2, \ldots, T
$$

where $\pi_{i t}$ is the $i$ th item moved by the crane in period $t$, and $\pi_{n_{t} t}$ is the last item to be moved by the crane in period $t$. Hence, the entire solution can be represented as

$$
\pi=\left\{\pi_{2}, \ldots, \pi_{T}\right\}=\left\{\left(\pi_{12}, \pi_{22}, \ldots, \pi_{n_{2} 2}\right),\left(\pi_{13}, \pi_{23}, \ldots, \pi_{n_{3} 3}\right), \ldots,\left(\pi_{1 T}, \pi_{2 T}, \ldots, \pi_{n_{T} T}\right)\right\} .
$$

Note in some cases, where temporary locations are used, the permutation may represent different possible solutions (different crane routes) with different cost unless a specific algorithm is used that leads to a unique crane route. Therefore, the serial method presented by McKendall et al. (2006) is used to obtain the actual crane routes.

To illustrate the CSP, its assumptions, and the above concepts, consider the CSP instance where the layout configuration and distance matrix $\left(d_{i j}\right)$ are shown in Figures 1 and 2, respectively. Also, the assignment of activities (and their required resources) to workspaces and the assignment of the idle resources to storage spaces are given in Figure 3. This CSP instance has 3 time periods $(T=3), 9$ resources $(R=9), 6$ locations $(L=6)$ and 4 activities. Also, each location has a capacity of 3 items $\left(C_{j}=3 \forall j\right)$. In period 1, activities 1 and 2 are performed in locations 1 and 2 (or workspaces 1 and 2), respectively. Since resources $(4)$ and $(2,3)$ are used to perform activities 1 and 2 , they are also assigned to locations 1 and 2 , respectively (i.e. $l_{41}=1, l_{21}=l_{31}=2$ ). In addition, idle resources $(1,8,9),(5,6)$ and $(7)$ are assigned to locations 4,5 and 6 , respectively (i.e. $\left.l_{11}=l_{81}=l_{91}=4, l_{51}=l_{61}=5, l_{71}=6\right)$. Therefore, the number of items in location 4 in period 1 is 3 (i.e. $n_{41}=3$ ). Notice activity 2 is also performed in both periods 1 and 2 at location 2, and activity 3 is performed at location 1 in period 2 . Therefore, at the beginning of period 2, location 2 is not available as a temporary location (i.e. $Q_{2}=\{2\}$ ), since activity 2 is still being performed there, but location 1 is available. All the items in bold font represent the items which are to be relocated at the beginning of periods 2 and 3. More specifically, items 1 and 4 are reassigned to locations 1 and 4 at the beginning of period 2 (i.e. $\left.A_{2}=\{1,4\}, B_{2}=\{1,4,10\}, l_{12}=1, l_{42}=4\right)$. In addition, items $(1),(2,3)$ and $(5,6)$ are reassigned to locations 6,5 and 2 , respectively, at the beginning of period 3 (i.e. $\left.A_{3}=\{1,2,3,5,6\}, B_{3}=\{1,2,3,5,6,10\}, l_{13}=6, l_{23}=l_{33}=5, l_{53}=l_{63}=2\right)$. As a result, the upper bound for the number of moves at period 3 is the product of 3 and the cardinality of the set $A_{3}$, which is $K=$ the maximum upper bound (i.e. $\left.K=U B_{3}=3\left|A_{3}\right|=3(5)=15\right)$. Therefore, the CSP determines the order in which the crane moves the items for each of the periods (periods 2 and 3 ) such that the sum of the travel and loading/unloading costs is minimised. 
Figure 1 Layout configuration for the crane sequencing problem instance

\begin{tabular}{|c|c|c|}
\hline $\begin{array}{c}\text { Work space 1 } \\
\text { (Location 1) }\end{array}$ & $\begin{array}{c}\text { Work space 2 } \\
\text { (Location 2) }\end{array}$ & $\begin{array}{c}\text { Work space 3 } \\
\text { (Location 3) }\end{array}$ \\
\hline $\begin{array}{c}\text { Storage space 1 } \\
\text { (Location 4) }\end{array}$ & $\begin{array}{c}\text { Storage space 2 } \\
\text { (Location 5) }\end{array}$ & $\begin{array}{c}\text { Storage space 3 } \\
\text { (Location 6) }\end{array}$ \\
\hline
\end{tabular}

Figure 2 Distance matrix $d_{i j}$ for the crane sequencing problem instance

\begin{tabular}{c|cccccc}
$i / j$ & 1 & 2 & 3 & 4 & 5 & 6 \\
\hline 1 & 0 & 1 & 2 & 1 & 2 & 3 \\
2 & 1 & 0 & 1 & 2 & 1 & 2 \\
3 & 2 & 1 & 0 & 3 & 2 & 1 \\
4 & 1 & 2 & 3 & 0 & 1 & 2 \\
5 & 2 & 1 & 2 & 1 & 0 & 1 \\
6 & 3 & 2 & 1 & 2 & 1 & 0
\end{tabular}

Figure 3 Item assignments for the crane sequencing problem instance

\begin{tabular}{|c|c|c|c|}
\hline \multirow[t]{2}{*}{$t=1$} & $4(A l)$ & $2,3(A 2)$ & \\
\hline & $1,8,9$ & 5,6 & 7 \\
\hline \multirow[t]{2}{*}{$t=2$} & $1(A 3)$ & $\mathbf{2 , 3}(A 2)$ & \\
\hline & $4,8,9$ & 5,6 & 7 \\
\hline \multirow[t]{2}{*}{$t=3$} & & $5,6(A 4)$ & \\
\hline & $4,8,9$ & 2,3 & 1,7 \\
\hline
\end{tabular}

As mentioned previously, there are multiple periods (i.e. $T=3$ ) considered in this CSP instance. As a result, the CSP will be used to generate $T-1$ crane routes (i.e. crane routes for periods 2 and 3). Also, the capacity of the crane is one unit of an item. Assume that the cost per distance unit is $\$ 5$ (i.e. $W_{1}=5$ ), and the cost of loading and unloading each item is $\$ 3$ (i.e. $W_{2}=3$ ). In addition, the initial location of the crane at the beginning of period 2 is location 1 (i.e. $p=1$ ) for this specific instance. At the beginning of period 2 , the crane needs to move items 1 and 4 to locations 1 and 4 , respectively. Hence, there are two (2!) possible sequences for the crane to relocate items 1 and 4 as shown in Table 1 . If the sequence of items to be moved is $1-4$ (i.e. $\pi_{2}=(1,4)$ ), the crane first needs to perform an empty move from its initial location (i.e. location 1) to the location of item 1 (i.e. location 4) which gives a travel cost of 1 distance unit. Once the crane arrives at location 4 , item 1 is loaded and prepared for moving. Next, the crane moves item 1 from locations 4 to 1 which gives a travel cost of 1 distance unit. Also, item 1 is unloaded. 
Therefore, loading/unloading cost for item 1 is $\$ 3$. Next, item 4 is considered to be moved, since it is the next item in the sequence. Since the crane is currently at location 1 where item 4 is located, item 4 is loaded and prepared for moving. Then, the crane moves item 4 from locations 1 to 4 , which gives a travel cost of 1 distance unit, and it is unloaded. As a result, loading/unloading cost of item 4 is $\$ 3$. Therefore, for this sequence, the total loading/unloading cost is $\$ 6$, and the total crane travel cost is $\$ 15$. Hence, the total cost of the sequence $1-4$ (i.e. $\pi_{2}=(1,4)$ ) is $\$ 21$. On the other hand, if the sequence of items to be moved is $4-1$ (i.e. $\pi_{2}=(4,1)$ ), the number of item moves is 3 and the crane travels 7 distance units. Therefore, the total cost of this sequence is $\$ 44$. More specifically, for this sequence, the crane starts at location 1 where item 4 is loaded and moved from location 1 to a temporary storage location 5 , since its destination location 4 is at full capacity. This is case (1) of temporary storage space usage. Initially, locations 1 , 3,5 and 6 can be used as temporary storage locations, since either no activity is currently being performed at these locations (unlike workspace 2) or the locations are not at full capacities (unlike location 4). However, since location 5 is closest to item 4's destination location (location 4), location 5 is selected as the temporary storage location. Ties are broken arbitrarily. This specific move gives a loading/unloading cost of $\$ 3$ and a crane travel cost of $\$ 10$. Then the crane moves from locations 5 to 4 (crane travel cost is $\$ 5$ ), item 1 is loaded and moved from locations 4 to 1 , and unloaded (crane travel cost $=\$ 5$ and loading/unloading cost $=\$ 3$ ). Now crane moves from locations 1 to 5 (crane travel cost $=\$ 10$ ), item 4 is loaded and moved to location 4 , and unloaded (crane travel cost $=\$ 5$ and loading/unloading cost $=\$ 3$ ). Thus, the sequence $4-1$ (i.e. $\left.\pi_{2}=(4,1)\right)$ gives the crane route 1-5-4-1-5-4 indicating the sequence in which the locations are visited by the crane. Nevertheless, sequence $1-4$ (i.e. $\pi_{2}=(1,4)$ ), which cost $\$ 21$, is much better than sequence $4-1$ (i.e. $\left.\pi_{2}=(4,1)\right)$, which cost $\$ 44$.

Table 1 The crane sequencing problem instance solutions at period $t=2$

\begin{tabular}{lccccc}
\hline Period & $\begin{array}{c}\text { Sequence of items } \\
\text { moved by the crane }\end{array}$ & $\begin{array}{c}\text { Actual moves of } \\
\text { the crane }\end{array}$ & $\begin{array}{c}\text { Distance units the } \\
\text { crane travels }\end{array}$ & $\begin{array}{c}\text { Number of } \\
\text { item moves }\end{array}$ & Cost \\
\hline $\boldsymbol{t}=\mathbf{2}$ & $\mathbf{1 - 4}$ & $\mathbf{1 - 4 - 1 - 4}$ & $\mathbf{3}$ & $\mathbf{2}$ & $\mathbf{\$ 2 1}$ \\
$t=2$ & $4-1$ & $1-5-4-1-5-4$ & 7 & 3 & $\$ 44$ \\
\hline
\end{tabular}

Table 2 The crane sequencing problem instance solutions at period $t=3$

\begin{tabular}{lccccc}
\hline Period & $\begin{array}{c}\text { Sequence of items } \\
\text { moved by the crane }\end{array}$ & $\begin{array}{c}\text { Actual moves of } \\
\text { the crane }\end{array}$ & $\begin{array}{c}\text { Distance units the } \\
\text { crane travels }\end{array}$ & $\begin{array}{c}\text { Number of } \\
\text { item moves }\end{array}$ & Cost \\
\hline$t=3$ & $1-2-5-3-6$ & $4-1-6-2-5-2-5-6$ & 10 & 5 & $\$ 65$ \\
$\boldsymbol{t}=\mathbf{3}$ & $\mathbf{1}-\mathbf{2}-\mathbf{5}-\mathbf{3}-\mathbf{6}$ & $\mathbf{4 - 1}-\mathbf{2}-\mathbf{5}-\mathbf{2}-\mathbf{5}-\mathbf{2}-\mathbf{6}$ & $\mathbf{8}$ & $\mathbf{6}$ & $\mathbf{\$ 5 8}$ \\
\hline
\end{tabular}

Table 3 Optimal solution for the crane sequencing problem instance

\begin{tabular}{lccccc}
\hline Period & $\begin{array}{c}\text { Sequence of items } \\
\text { moved by the crane }\end{array}$ & $\begin{array}{c}\text { Actual moves of } \\
\text { the crane }\end{array}$ & $\begin{array}{c}\text { Distance units } \\
\text { the crane travels }\end{array}$ & $\begin{array}{c}\text { Number of } \\
\text { item moves }\end{array}$ & Cost \\
\hline$t=2$ & $1-4$ & $1-4-1-4$ & 3 & 2 & $\$ 21$ \\
$t=3$ & $1-2-5-3-6$ & $4-1-2-5-2-5-2-6$ & 8 & 6 & $\$ 58$ \\
& & & & & $\$ 79$ \\
\hline
\end{tabular}


For period 3, the initial location of the crane is the location of the last position of the crane in period 2. As a result, the crane is located at location 4. Also, the number of possible crane sequences is $5 !=120$. In Table 2 , one of the crane sequences is considered to illustrate temporary storage location issues, specifically, how different crane routes are formed from the same sequence. Consider crane sequence $1-2-5-3-6$ (i.e. $\pi_{3}=(1,2,5$, $3,6)$ ). First, the crane moves empty from locations 4 to 1 (crane travel cost $=\$ 5$ ). Second, item 1 is loaded and moved from locations 1 to 2 (crane travel cost $=\$ 5$ and loading/unloading cost $=\$ 3$ ), since using location 2 as temporary storage space reduces total cost, which considers case (2) above for temporary storage space usage. Next, items 2, 5, 3 and 6 are loaded and moved in order from locations 2, 4, 2 and 4 to their assigned locations 4, 2, 4 and 2, respectively (crane travel cost $=\$ 20$ and loading/unloading cost $=\$ 12$ ). Because all other items had been moved to their destination locations, item 1 is loaded and moved from its temporary location (location 2) to its required location (location 6) and is unloaded (crane travel cost $=\$ 10$ and loading/unloading cost $=\$ 3$ ). Therefore, the cost of sequence $1-2-5-3-6$ (i.e. $\left.\pi_{3}=(1,2,5,3,6)\right)$ is $\$ 58$. However, the sequence does not indicate when an item is moved to/from a temporary storage location, and more than one crane route may be obtained for a specific sequence. More specifically, the sequence $1-2-5-3-6$ (i.e. $\left.\pi_{3}=(1,2,5,3,6)\right)$ yields a crane route 4-1-2$5-2-5-2-6$ with total cost of $\$ 58$. For the same sequence, a crane route of 4-1-6-2-5-2$5-6$ may be obtained with a total cost of $\$ 65$. Notice in this crane route, item 1 is moved directly to its destination location. Therefore, using temporary storage location can reduce total cost. Recall, the serial method presented by McKendall et al. (2006) is used to obtain an efficient crane route given a sequence.

In Table 3, the optimal solution for this CSP instance is given as the following sequences for periods 2 and $3: 1-4$ and $1-2-5-3-6$, respectively (i.e. $\pi=\left\{\pi_{2}, \pi_{3}\right\}=\{(1$, $4),(1,2,5,3,6)\}$. The total cost of this solution is $f(\pi)=\$ 79=\$ 21+\$ 58$. The optimal solution was obtained using the binary integer linear program presented above for the CSP and the CPLEX solver, Version 6.6. It took 0.1 minutes on a Pentium IV $2.8 \mathrm{GHz}$ PC. However, for another small CSP with 9 items and 4 periods, it required 8.1 hours of computation time. Therefore, only small-size CSPs are solved in reasonable computation time using the mathematical model. As a result, HASs are presented next to solve largesize CSPs in reasonable time.

\section{Methodology}

A local neighbourhood search technique starts with an initial solution $\pi$, as the current solution, and randomly selects a neighbouring solution $\pi^{\prime}$ in the neighbourhood of $\pi$, $\pi^{\prime} \in N(\pi)$. A neighbouring solution $\pi^{\prime}$ is obtained by randomly selecting a period $t$ $(1<t \leq T)$ and randomly exchanging the locations of two items in $\pi_{t}$. If $f\left(\pi^{\prime}\right)<f(\pi)$, then $\pi^{\prime}$ becomes the current solution (set $\pi=\pi^{\prime}$ ). Otherwise, the current solution $\pi$ does not change. This process is repeated until a predefined stopping criterion has been met. This technique is known as the random descent heuristic. Since only improving solutions are accepted during the search process, this local search technique as well as others (e.g. steepest descent, first improvement), converge to the local optimum of the initial solution. Often times, this may result in a low-quality solution. Hence, this method does not guarantee a global optimum, but it is used within many meta-heuristics such as the simulated annealing and HASs which are used to improve the performances of simple 
local neighbourhood search techniques. More specifically, the random descent heuristic is used to search the neighbourhoods of solutions, and other components of the proposed HASs will be used to accept non-improving solutions so that the global optimum may be obtained. Next, the proposed HASs are presented for the CSP.

Gambardella, Taillard and Dorigo (1999) presented an ant system optimisation technique, called HAS, for the quadratic assignment problem (HAS-QAP). It is used to improve a set of randomly generated solutions. HAS is a modification of the ant colony optimisation (ACO) heuristics presented in Colorni, Dorigo and Maniezzo (1991) and Dorigo and Gambardella (1997). In both papers, the ACO heuristics were used to construct solutions for the travelling salesman problem (TSP). Dorigo et al., (1996) discuss the applications of ant systems to construct solutions for some well-known problems such as the TSP and vehicle routing problem. Several authors presented ant systems for the quadratic assignment problem (QAP) such as Gambardella, Taillard and Dorigo (1999), Maniezzo and Colorni (1999) and Stutzle and Dorigo (1999). The ACO and HAS heuristics use the idea of how ants search for food and leave a chemical substance called pheromone so that other ants can find the food source to solve combinatorial optimisation problems. The pheromone trails in these ant systems serve as distributed, numerical information which the ants use to probabilistically construct (as in ACO) or improve (as in HAS) solutions to combinatorial optimisation problems.

The artificial ants in the HAS represent solutions which are stochastically modified based on the pheromone trail matrix (i.e. entries in matrix represent the desirability of moving item $r$ in the $j$ th position in period $t$ ). The HAS also provides mechanisms to either intensify or diversify the search. McKendall and Shang (2006) modified the HASQAP heuristic and presented three HASs for the dynamic facility layout problem (multiperiod QAP). The first heuristic is a direct modification of HAS-QAP for the dynamic facility layout problem. The second heuristic uses a simulated annealing heuristic, instead of a random descent heuristic as in HAS-QAP, to improve the solutions obtained after performing pheromone trail swaps. The third heuristic is exactly like the first, except that the random descent heuristic has a look-ahead/look-back strategy. In this article, two HASs are presented for the CSP. The first is similar to the HAS-QAP (use random descent heuristic as a local search technique), and the second uses simulated annealing instead of a random descent heuristic. The first HAS, called HAS I, is briefly summarised below.

Step 1. Set parameters and counters:

$M=$ number of ants (solutions);

$Q=$ parameter used to determine the initial values of the pheromone trail matrix $P$;

$E=$ number of pheromone trail exchanges used to modify each CSP solution;

$q=$ the probability that pheromone trail exchange policy 1 will be selected to modify a CSP solution. As a result, policy 2 is chosen with a probability of $1-q$; the policies are discussed below.

$\alpha_{1}, \alpha_{2}=$ parameters used to weaken and enforce pheromone trails, respectively;

$S=$ number of consecutive iterations, without improving best solution $\pi^{*}$, before implementing diversification strategy;

Set intense $=1$ (intensification strategy is active); 
run_time $=$ run time of heuristic;

Set trail swap counter $e=0$;

Set iteration counter $i t=0$;

Set iteration without improvement counter $i t w=0$;

Step 2. Randomly generate a set of initial CSP solutions, called $\Omega$. That is, list items to be moved in random order in each period for each ant, $\pi^{m}$, where $m=1,2, \ldots, M$ and $\pi^{m} \in \Omega$.

Step 3. Use the random descent method presented above to improve all $M$ solutions $\pi^{m} \in \Omega$. The set of improved solutions are defined as $\hat{\Omega}$ (i.e. $\hat{\pi}^{m} \in \hat{\Omega}$ ).

Set $f\left(\pi^{*}\right)=\min \left\{f\left(\hat{\pi}^{m}\right) \mid \forall m\right\}$.

Step 4. Initialise pheromone trail matrix $P$ :

Set entries of $P, p_{r j t}=Q / f\left(\pi^{*}\right) \quad \forall r \in A_{t}, j, t$, where the entries $p_{r j t}$ measures the desirability of assigning item $r=\hat{\pi}_{i t}^{m}$ (item $r$ in $i$ th position in period $t$ ) to be moved to the $j$ th position in $\hat{\pi}_{t}^{m}$ of $\hat{\pi}^{m}$ and $A_{t}$ is a set of items to be moved.

Step 5. Modify all $\hat{\pi}^{m} \in \hat{\Omega}$ by using the pheromone trail matrix and one of two policies to obtain another set of solutions $\bar{\Omega}$ (i.e. $\bar{\pi}^{m} \in \bar{\Omega}$ ). More specifically, for each $\hat{\pi}^{m}$ : Set counter $e=1$;

Step 5a):

If $e<E$,

Randomly select a period $t$ and an item $x \in \hat{\pi}_{t}^{m}$, say $\hat{\pi}_{u t}^{m}$;

Randomly generate a number $w$ between 0 and 1;

If $w \leq q$,

Then use policy 1. That is, select a new position $v \neq u$ for item $x$ such that $p_{x v t}+p_{y u t}$ is maximised where item $y$ is currently in position $v$ in period $t$ (i.e. $\left.y=\hat{\pi}_{v t}^{m}\right)$;

Else,

Use policy 2. That is, $v \neq u$ is chosen with a probability of $p_{x v t}+p_{y u t} / \sum_{v \neq u}\left(p_{x v t}+p_{y u t}\right)$ where $y$ is currently in position $v$ in period $t$ (i.e. $\left.y=\hat{\pi}_{v t}^{m}\right)$;

Exchange the locations of items $x$ and $y$ in $\hat{\pi}_{t}^{m}$;

Set $e=e+1$, and go to the beginning of Step 5a);

Else, $E$ trail swaps have been performed;

After all solutions $\hat{\pi}^{m} \in \hat{\Omega}$ have been modified such that the new set of solutions $\bar{\Omega}$ is obtained, go to Step 6 .

Step 6. Use the random descent heuristic to improve solutions $\bar{\pi}^{m}$ in the set $\bar{\Omega}$ and obtain the set of solutions $\tilde{\Omega}\left(\tilde{\pi}^{m} \in \tilde{\Omega}\right)$.

If $\exists \tilde{\pi}^{m} \in \tilde{\Omega}$ such that $f\left(\tilde{\pi}^{m}\right)<f\left(\pi^{*}\right)$, then

set $\pi^{*}=\tilde{\pi}^{m}$ where $f\left(\pi^{*}\right)=\min \left\{f\left(\tilde{\pi}^{m}\right) \mid \tilde{\pi}^{m} \in \tilde{\Omega}\right\}$; 
set $i t w=0$;

Else, $i t w=i t w+1$;

Step 7. If $i t w=S$, set $i t w=0$, and randomly generate a set of $M-1$ solutions. The $M-1$ solutions and $\pi^{*}$ make up a diverse set of solutions define as $\Omega$. Then go to Step 3;

Step 8. If intense $=1$,

Set temp $=0$;

For each $m$, if $f\left(\hat{\pi}^{m}\right)>f\left(\tilde{\pi}^{m}\right)$,

set $\hat{\pi}^{m}=\tilde{\pi}^{m}$ and temp $=1$;

Set intense $=$ temp;

Else, set $\hat{\Omega}=\tilde{\Omega}$.

If $i t w=0$, then set intense $=1$;

Step 9. Update pheromone trail matrix as discussed below.

Step 10. If heuristic run time $>$ run_time, then terminate the heuristic.

Else, go to Step 5.

In Step 3, the randomly generated solutions obtained in Step 2 are improved using the random descent heuristic. Based on the value of the best solution obtained in Step 3, the pheromone trail matrix is initialised in Step 4. In Step 5, a period $t$ and an item $x$ in position $u$ are randomly selected. The order in which the crane will move this item is exchanged with another item $y$ in position $v$ which is selected based on one of two policies. The first policy uses the pheromone trail matrix to select item $y$ in location $v$ based on the most desirable exchange between item $x$ in position $u$ and all other items. The second policy selects an item $y$ in location $v$ randomly, but there is a higher probability that item $y$ with the largest $p_{x v t}+p_{y u t}$ will be accepted. After $E$ exchanges using the pheromone trail matrix, the solutions are improved using the random descent heuristic in Step 6. If $S$ iterations are performed without improving the best found solution, the diversification strategy is implemented in Step 7. This strategy is used to explore diverse areas of the solution space by taking the best solution found $\pi^{*}$, randomly generating $M-1$ solutions to obtain a different set of solutions $(\Omega)$, and continuing the heuristic at Step 3. Otherwise, the intensification strategy is invoke in Step 8, if it is active (i.e. intense $=1$ ). If intense $=0$, then the intensification strategy is not active. Intensification is used to explore promising areas of the solution space. In other words, the set of solutions $\hat{\Omega}$ for the next iteration is obtained based on the intensification strategy. More specifically, initially the intensification strategy is activated, which means that the better solution between $\hat{\pi}^{m}$ and $\tilde{\pi}^{m}$ is used as the starting solution at the next iteration $\left(\hat{\pi}^{m}\right)$ for each solution $m$. This strategy remains active if at least one solution $m$ is improved during an iteration. Otherwise, the intensification strategy is inactive, and the solution at the next iteration for each ant is $\tilde{\pi}^{m}$ (i.e. $\hat{\pi}^{m}=\tilde{\pi}^{m} \forall m$ ). If the strategy is inactive, then it becomes active when the best solution $\pi^{*}$ is improved. In step 9 , the pheromone trail matrix $P$ is updated such that only the best solution found so far is permitted to deposit pheromone. The pheromone trail matrix is updated according to Dorigo and Gambardella (1997). Set 


$$
p_{r j t}=\left(1-\alpha_{1}\right) p_{r j t}+\alpha_{2} \delta\left(\pi_{r j t}\right) \quad \forall r \in A_{t}, j, t
$$

where

$$
\delta\left(\pi_{r j t}\right)=\left\{\begin{array}{ll}
1 / f\left(\pi^{*}\right) & \text { if } \pi_{r j t} \in \text { best solution } \pi^{*} \\
0 & \text { otherwise }
\end{array} .\right.
$$

The first term in the expression, $\left(1-\alpha_{1}\right) p_{r j t}$, is used to weaken the pheromone trails where $0<\alpha_{1}<1$ is a parameter used to control the evaporation rate of the trails. A value close to zero indicates the trails will remain active for a longer time, and a value close to one indicates a high degree of evaporation and a shorter memory of the system. However, the expression $\alpha_{2} \delta\left(\pi_{r j t}\right)$ is used to strengthen the pheromone trails where $\alpha_{2}$ is a parameter used to control the reinforcement of the pheromone trails corresponding to the best solution $\pi^{*}$. Using only the best solution $\pi^{*}$ speeds up the convergence of the heuristic (Gambardella, Taillard and Dorigo, 1999). In step 10, the heuristic is terminated, if heuristic run time is at least run time minutes.

The second HAS, called HAS II, uses a simulated annealing heuristic instead of a random descent heuristic, to improve solutions initially and after performing $E$ pheromone trail exchanges. McKendall and Shang (2006) presented a similar HAS for the dynamic facility layout problem, which out-performed the HAS with a random descent heuristic. Therefore, it is applied to the CSP presented in this article. More specifically, the random descent heuristic presented in Steps 3 and 6 is replaced by the following simulated annealing heuristic.

Step 1. Set parameters and counters:

$T_{0}=$ initial temperature;

$\epsilon=$ cooling ratio $(\epsilon=0.95)$;

$H_{0}=$ number of iterations performed at initial temperature epoch length, $\left(H_{0}=T\right.$ where $T=$ number of periods);

$\gamma=$ parameter used to increase epoch length $(\gamma=0.04)$;

$T_{\mathrm{f}}=$ final temperature $\left(T_{\mathrm{f}}=0.1\right)$;

$\pi^{m}=$ current solution;

$\pi^{*}=$ best solution found;

Set current temperature Temp $=T_{0}$;

Set current epoch length $H=H_{0}$;

Set iteration counter at each temperature $i c=0$;

Set $\pi^{*}=\pi^{m}$;

Step 2. Set $i c=i c+1$;

Generate a neighbouring solution $\pi^{\prime}$ in the neighbourhood of current solution $\pi^{m}$ by random pairwise exchange (i.e. $\pi^{\prime} \in N\left(\pi^{m}\right)$ ).

Step 3. If $f\left(\pi^{\prime}\right) \leq f\left(\pi^{*}\right)$, then set $\pi^{*}=\pi^{\prime}, \pi^{m}=\pi^{\prime}$, and go to step 4;

Else,

if $f\left(\pi^{\prime}\right) \leq f\left(\pi^{m}\right)$, then set $\pi^{m}=\pi^{\prime}$;

Else, if $\operatorname{rand}(0,1)<\exp \left(-\left[f\left(\pi^{\prime}\right)-f\left(\pi^{m}\right)\right] /\right.$ Temp $)$, set $\pi^{m}=\pi^{\prime}$; 
Step 4. If $i c<H$, then go to step 2;

Else, set Temp $=\epsilon$ Temp and $H=H(1+\gamma)$;

If Temp $>T_{f}$, set $i c=0$ and go to step 2;

Else, terminate heuristic.

This simulated annealing heuristic is repeated for each ant. First, the temperature Temp has been set to the initial value $T_{0}$ at the beginning. The initial temperature $T_{0}$ was determined by using the formula $\exp \left(-\left[f\left(\pi^{\prime}\right)-f\left(\pi^{m}\right)\right] / T_{0}\right)=0.25$ and $f\left(\pi^{\prime}\right)-f\left(\pi^{m}\right)=0.1^{*} f\left(\pi^{m}\right)$, where 0.10 and 0.25 were obtained experimentally. In other words, $T_{0}=-0.10 * f\left(\pi^{m}\right) / \ln (0.25)$ and the initial probability of accepting a nonimproving solution, which is $10 \%$ worse then solution $\pi^{m}$, is $25 \%$. Then, Temp is decreased by $\epsilon$ Temp (i.e. Temp $=\epsilon$ Temp) at every temperature reduction. In other words, after performing $H$ number of random pairwise exchanges, the temperature is reduced by єTemp. The epoch length $H$ is the number of iterations (random pairwise exchanges) performed at each temperature. At the initial temperature $T_{0}$, the number of random pairwise exchanges is the number of periods (i.e. $H_{0}=T$ ). Otherwise, the number of random pairwise exchanges is $H(1+\gamma)$, as in the simulated annealing heuristic presented by Bouleimen and Lecocq (2003) for the resource constrained project scheduling problem. The process is continued until a predefined stopping temperature has been met (i.e. Temp $\leq T_{f}$ ). Initially, the probability of allowing a non-improved move, $\exp \left(-\left[f\left(\pi^{\prime}\right)-f\left(\pi^{m}\right)\right] /\right.$ Temp $)$ will be relatively large. However, as Temp decreases, this probability also decreases. Furthermore, $\epsilon, \gamma$ and $H_{0}$ were set experimentally.

\section{Computational experiments and analysis}

Two test data sets are presented in this article. Data set 1 is randomly generated based on the following factors: the total number of items, $R$; the number of periods, $T$ and the number of locations, $L$. Hence, there are $L / 2$ workspace locations and $L / 2$ storage space locations and distance between any two locations $i$ and $j$ are defined in a distance matrix $D$. The number of items to be moved (NOIM) is uniformly distributed in $[1, R]$; the number of destination locations (NODL) is uniformly distributed in [1, NOIM], and the number of items in destination location is uniformly distributed in [1, Cap]. For instance, if $\mathrm{NOIM}=3$ for a specific period $t$, then NODL is uniformly distributed in $[1,3]$, say 2 . Therefore, 3 items in period $t$ is moved and assigned to two locations. First, randomly generate 2 locations uniformly distributed in $[1, L]$. Next, randomly assign the 3 items to two locations. This is done for each period $t$. In the first data set, the number of items $R$ is 9 , the number of locations $L$ is 6 and the number of periods $T$ is from 2 to 7 as shown in Table 4.

Data set 2 was obtained by solving the dynamic space allocation problem (DSAP) instances presented in McKendall et al., (2005). The DSAP assigns activities and their required items (resources) to workspaces and idle items to storage spaces with respect to minimising the sum of the distances the items travel. Therefore, the output of the DSAP can be used as input data for the CSP. This data set has 96 test problems. The sizes of the instances in this data set are shown in Table 5. 
For HAS I and II heuristics, the following parameters setting are used. The numbers of ants $(M)$ are set to 5, 10, 12 and 15 for group 1 (i.e. data set 1 and all problems with $R=9$ in data set 2), 2 (i.e. all problems with $R=18$ in data set 2), 3 (i.e. all problems with $R=30$ in data set 2) and 4 (i.e. all problems with $R=48$ in data set 2), respectively. $E=R * T, \alpha_{1}=0.1$ and $\alpha_{2}=Q=T^{*} 10^{3}, q=0.7, S=R * T$. Furthermore, the settings for the simulated annealing heuristic embedded within HAS II are $T_{0}=-0.10^{*} f\left(\pi^{m}\right) / \ln (0.25)$, as well as $\epsilon, \gamma, T_{\mathrm{f}}$ and $H_{0}$ were set to $0.95,0.04,0.1$ and number of periods $(T)$, respectively.

In all experiments, a Pentium IV $2.4 \mathrm{GHz}$ PC was used to solve the CSP instances from data sets I and II using the proposed heuristics. The heuristics were coded using the $\mathrm{C}++$ programming language under Windows XP operating system. The proposed HAS heuristics were compared with the simulated annealing heuristic presented in McKendall et al. (2006) except that the objective function includes both crane travel cost and loading/unloading cost. In order to make fair comparisons, the proposed HAS and simulated annealing heuristics ran for the same amount of computational time for each CSP problem instance.

Table 4 Problem sizes for data set 1

\begin{tabular}{llll}
\hline Pr. number & $R$ & $L$ & $T$ \\
\hline P01-P04 & 9 & 6 & 2 \\
P05-P08 & 9 & 6 & 3 \\
P09-P12 & 9 & 6 & 4 \\
P13-P16 & 9 & 6 & 5 \\
P17-P20 & 9 & 6 & 6 \\
P21-P24 & 9 & 6 & 7 \\
\hline
\end{tabular}

Table 5 Problem sizes for data set 2

\begin{tabular}{lrrl}
\hline Pr. number & $R$ & $L$ & $T$ \\
\hline P01-P08 & 9 & 6 & 10 \\
P09-P16 & 9 & 6 & 15 \\
P17-P24 & 9 & 6 & 20 \\
P25-P32 & 18 & 12 & 10 \\
P33-P40 & 18 & 12 & 15 \\
P41-P48 & 18 & 12 & 20 \\
P49-P56 & 30 & 20 & 10 \\
P57-P64 & 30 & 20 & 15 \\
P65-P72 & 30 & 20 & 20 \\
P73_P80 & 48 & 32 & 10 \\
P81-P88 & 48 & 32 & 15 \\
P89-P96 & 48 & 32 & 20 \\
\hline
\end{tabular}


Table 6 Computational results for generated data set 1

\begin{tabular}{|c|c|c|c|c|c|}
\hline \multirow[b]{2}{*}{ Pr. number } & \multicolumn{2}{|c|}{ Optimal solution } & \multirow[b]{2}{*}{ Simulated annealing } & \multirow[b]{2}{*}{$H A S I / I I$} & \multirow[b]{2}{*}{ Heuristic time } \\
\hline & Final & Time & & & \\
\hline$\overline{\text { P01 }}$ & 57 & 0.01 & 57 & 57 & 0.00 \\
\hline P02 & 83 & 0.05 & 83 & 83 & 0.00 \\
\hline P03 & 62 & 0.03 & 62 & 62 & 0.00 \\
\hline P04 & 88 & 0.11 & 88 & 88 & 0.01 \\
\hline P05 & 65 & 0.08 & 65 & 65 & 0.01 \\
\hline P06 & 119 & 7.60 & 119 & 119 & 0.01 \\
\hline P07 & 72 & 0.02 & 72 & 72 & 0.01 \\
\hline P08 & 91 & 0.23 & 91 & 91 & 0.02 \\
\hline P09 & 121 & 0.33 & 121 & 121 & 0.02 \\
\hline P10 & 150 & 19.42 & 150 & 150 & 0.02 \\
\hline P11 & 118 & 2.02 & 118 & 118 & 0.02 \\
\hline P12 & 150 & 19.53 & 150 & 150 & 0.01 \\
\hline P13 & 104 & 0.10 & 104 & 104 & 0.01 \\
\hline P14 & 189 & 1527.36 & 189 & 189 & 0.02 \\
\hline P15 & 114 & 0.12 & 114 & 114 & 0.02 \\
\hline P16 & 229 & 8.48 & 229 & 229 & 0.02 \\
\hline P17 & 145 & 0.12 & 145 & 145 & 0.02 \\
\hline P18 & 278 & 1947.32 & 278 & 278 & 0.02 \\
\hline P19 & 157 & 60.97 & 157 & 157 & 0.02 \\
\hline P20 & 246 & 1014.73 & 246 & 246 & 0.03 \\
\hline P21 & 169 & 2.93 & 169 & 169 & 0.03 \\
\hline P22 & 310 & 2915.87 & 310 & 310 & 0.04 \\
\hline P23 & 197 & 318.12 & 197 & 197 & 0.04 \\
\hline P24 & 347 & 2755.59 & 347 & 347 & 0.03 \\
\hline
\end{tabular}

The optimal solutions in Table 6 were obtained for all of the test problems in data set 1 and were obtained using the mathematical model presented previously and the CPLEX solver, Version 6.6. The following CPLEX parameter settings were used: strong branching for variable selection, depth first for node selection, dual simplex for start algorithm and the rest were default settings. Also, their computational times are listed. The bold numbers represent the optimal objective function values (OFVs). All times in the tables are given in minutes. As a result, both HAS and simulated annealing heuristics obtained the optimal solutions for all 24 test problems. Notice the run times for the heuristics are much less than the run times for the mathematical model with CPLEX. The main reason for using this data set was to test the mathematical model.

The second data set is used to further verify the speed and robustness of the proposed heuristics. This data set has test problems with 9-48 items, 6-32 locations 10, 15 and 20 periods as shown in Table 5. Compared to data set 1 , data set 2 is much larger and has much larger problems, which cannot be solved by using exact methods in reasonable time. simulated annealing, HAS I and II, ran 15 times each with the same randomly generated initial solutions. Data set 2 is divided into four groups, each of which has 
24 test problems. From groups 1 to 4 , the sizes of the test problems are increased. The test problems in group 1 are relative small, groups 2 and 3 have medium size test problems, and the test problems in group 4 are the largest.

For data set 2, Table 7 shows the number of best solutions obtained by each of the proposed heuristics, and Table 8 gives the average percent each heuristic is away from best found solutions. For the first group (i.e. test problems 01-24), the test problems have 9 resources, 6 locations and 3-5 periods, which are the smaller size problems. All heuristics performed well for this group of test problems. More specifically, HAS I obtained the best found solution 23 times, HAS II, 22 times, and simulated annealing 23 times. The detail results for the first group are given in Table 9. Note, the bolded OFVs are the best found OFVs.

However, the HAS and simulated annealing heuristics perform differently for the other three groups of test problems. More specifically, HAS I obtained the best found solution 12, 7 and 6 times, HAS II obtained best 12, 16 and 17 times, and simulated annealing obtained best 16, 3 and 5 times. See the results for each test problem in groups 2,3 and 4 in Tables 10,11 and 12, respectively.

Overall in Table 7, HAS II outperformed the other two heuristics since it obtained 67 best solutions of 96 test problems, which is the most. Moreover, HAS I performed slightly better than simulated annealing since HAS I obtained best found 48 times and simulated annealing obtained best for 47 of 96 test problems. Since this information may be misleading, the average percent each heuristic is away from the best found solution is given in Table 8. More specifically, the average percent the HAS II, HAS I and simulated annealing heuristics are above the best found solutions are $0.20,0.28$ and $0.74 \%$, respectively. In other words, although simulated annealing and HAS I obtained about the same number of best found solutions, HAS I obtained solutions closer to the best found than simulated annealing, on average.

Tables 9-12 list detail results and computational times for each group of test problems in data set 2 . The bold numbers are the best found OFVs, and times are given in minutes. Recall, all heuristics ran for the same amount of time with 15 runs. Although the HAS II heuristic is a combination of the HAS I with simulated annealing, it outperformed the HAS I and simulated annealing heuristics with the same run times. Although this integration of heuristics increases the computational complexity, the results show that HAS II improved HAS I. In other words, even though an iteration in the HAS II require more computation time, less iterations need to be performed to obtain superior solutions. Hence, HAS II is the preferred heuristic.

Table 7 Number of best solutions found obtained by the proposed heuristics

\begin{tabular}{lccc}
\hline No & $H A S I$ & HASII & Simulated annealing \\
\hline P01-P24 & 23 & 22 & 23 \\
P25-P48 & 12 & 12 & 16 \\
P49-P72 & 7 & 16 & 3 \\
P73-P96 & 6 & 17 & 5 \\
Total & 48 & 67 & 47 \\
Percent & 50.0 & 69.8 & 49.0 \\
\hline
\end{tabular}


Table 8 Average percent each heuristic is away from the best found solutions

\begin{tabular}{cccc}
\hline No & HASI \% & HASII \% & Simulated annealing \% \\
\hline P01-P24 & 0.09 & 0.12 & 0.09 \\
P25-P48 & 0.45 & 0.34 & 0.42 \\
P49-P72 & 0.32 & 0.21 & 1.35 \\
P73-P96 & 0.27 & 0.14 & 1.11 \\
Average & 0.28 & 0.20 & 0.74 \\
\hline
\end{tabular}

Table 9 Computational results for data set 2 with $R=9$ and $L=6$

\begin{tabular}{|c|c|c|c|c|c|c|c|}
\hline \multirow[b]{2}{*}{ No } & \multirow[b]{2}{*}{ Initial cost } & \multicolumn{3}{|c|}{$H A S$} & \multirow[b]{2}{*}{ Simulated annealing } & \multirow[b]{2}{*}{ Best of all } & \multirow[b]{2}{*}{ Time } \\
\hline & & $H A S I$ & HASII & Best & & & \\
\hline 1 & 205 & 205 & 205 & 205 & 205 & 205 & 0.03 \\
\hline 2 & 359 & 339 & 339 & 339 & 339 & 339 & 0.05 \\
\hline 3 & 243 & 233 & 233 & 233 & 233 & 233 & 0.03 \\
\hline 4 & 324 & 314 & 314 & 314 & 314 & 314 & 0.05 \\
\hline 5 & 213 & 213 & 213 & 213 & 213 & 213 & 0.03 \\
\hline 6 & 385 & 375 & 375 & 375 & 375 & 375 & 0.05 \\
\hline 7 & 274 & 254 & 254 & 254 & 254 & 254 & 0.05 \\
\hline 8 & 369 & 346 & 346 & 346 & 346 & 346 & 0.03 \\
\hline 9 & 410 & 390 & 390 & 390 & 390 & 390 & 0.07 \\
\hline 10 & 655 & 645 & 645 & 645 & 645 & 645 & 0.07 \\
\hline 11 & 462 & 442 & 442 & 442 & 442 & 442 & 0.07 \\
\hline 12 & 720 & 637 & 647 & 637 & 637 & 637 & 0.07 \\
\hline 13 & 403 & 373 & 373 & 373 & 373 & 373 & 0.05 \\
\hline 14 & 595 & 585 & 585 & 585 & 585 & 585 & 0.05 \\
\hline 15 & 566 & 460 & 460 & 460 & 460 & 460 & 0.07 \\
\hline 16 & 733 & 670 & 670 & 670 & 670 & 670 & 0.07 \\
\hline 17 & 467 & 467 & 467 & 467 & 467 & 467 & 0.08 \\
\hline 18 & 856 & 806 & 806 & 806 & 806 & 806 & 0.08 \\
\hline 19 & 651 & 621 & 621 & 621 & 621 & 621 & 0.10 \\
\hline 20 & 1,031 & 938 & 918 & 918 & 938 & 918 & 0.08 \\
\hline 21 & 711 & 615 & 615 & 615 & 615 & 615 & 0.08 \\
\hline 22 & 926 & 853 & 853 & 853 & 853 & 853 & 0.08 \\
\hline 23 & 848 & 692 & 702 & 692 & 692 & 692 & 0.10 \\
\hline 24 & 981 & 786 & 786 & 786 & 786 & 786 & 0.08 \\
\hline
\end{tabular}


Table 10 Computational results for data set 2 with $R=18$ and $L=12$

\begin{tabular}{|c|c|c|c|c|c|c|c|}
\hline \multirow[b]{2}{*}{ No } & \multirow[b]{2}{*}{ Initial cost } & \multicolumn{3}{|c|}{$H A S$} & \multirow[b]{2}{*}{ Simulated annealing } & \multirow[b]{2}{*}{ Best of all } & \multirow[b]{2}{*}{ Time } \\
\hline & & $H A S I$ & $H A S I I$ & Best & & & \\
\hline 25 & 610 & 490 & 490 & 490 & 490 & 490 & 0.45 \\
\hline 26 & 813 & 632 & 622 & 622 & 622 & 622 & 0.43 \\
\hline 27 & 982 & 513 & 513 & 513 & 513 & 513 & 0.50 \\
\hline 28 & 1,038 & 730 & 730 & 730 & 730 & 730 & 0.47 \\
\hline 29 & 544 & 441 & 441 & 441 & 441 & 441 & 0.43 \\
\hline 30 & 783 & 660 & 660 & 660 & 660 & 660 & 0.45 \\
\hline 31 & 791 & 557 & 537 & 537 & 537 & 537 & 0.48 \\
\hline 32 & 1,167 & 827 & 837 & 827 & 824 & 824 & 0.48 \\
\hline 33 & 1,107 & 794 & 794 & 794 & 794 & 794 & 0.70 \\
\hline 34 & 1,434 & 1,065 & 1,065 & 1,065 & 1,065 & 1,065 & 0.70 \\
\hline 35 & 1,249 & 856 & 880 & 856 & 896 & 856 & 0.77 \\
\hline 36 & 1,670 & 1,214 & 1,212 & 1,212 & 1,193 & 1,193 & 0.73 \\
\hline 37 & 895 & 687 & 682 & 682 & 692 & 682 & 0.68 \\
\hline 38 & 1,312 & 1,085 & 1,082 & 1,082 & 1,079 & 1,079 & 0.70 \\
\hline 39 & 1,276 & 819 & 817 & 817 & 827 & 817 & 0.75 \\
\hline 40 & 1,793 & 1,241 & 1,238 & 1,238 & 1,229 & 1,229 & 0.75 \\
\hline 41 & 1,587 & 1,159 & 1,160 & 1,159 & 1,171 & 1,159 & 0.93 \\
\hline 42 & 1,844 & 1,408 & 1,409 & 1,408 & 1,409 & 1,408 & 0.95 \\
\hline 43 & 1,765 & 1,275 & 1,273 & 1,273 & 1,272 & 1,272 & 1.05 \\
\hline 44 & 2,566 & 1,864 & 1,858 & 1,858 & 1,860 & 1,858 & 1.03 \\
\hline 45 & 1,326 & 1,016 & 1,014 & 1,014 & 1,013 & 1,013 & 0.90 \\
\hline 46 & 1,798 & 1,457 & 1,457 & 1,457 & 1,455 & 1,455 & 0.93 \\
\hline 47 & 1,958 & 1,291 & 1,296 & 1,291 & 1,313 & 1,291 & 1.05 \\
\hline 48 & 2,562 & 1,828 & 1,835 & 1,828 & 1,830 & 1,828 & 1.02 \\
\hline
\end{tabular}

Table 11 Computational results for data set 2 with $R=30$ and $L=20$

\begin{tabular}{|c|c|c|c|c|c|c|c|}
\hline \multirow[b]{2}{*}{ No } & \multirow[b]{2}{*}{ Initial cost } & \multicolumn{3}{|c|}{$H A S$} & \multirow[b]{2}{*}{ Simulated annealing } & \multirow[b]{2}{*}{ Best of all } & \multirow[b]{2}{*}{ Time } \\
\hline & & HASI & HASII & Best & & & \\
\hline 49 & 1,085 & 816 & 802 & 802 & 812 & 802 & 2.82 \\
\hline 50 & 1,210 & 936 & 943 & 936 & 951 & 936 & 2.87 \\
\hline 51 & 1,162 & 799 & 798 & 798 & 809 & 798 & 3.12 \\
\hline 52 & 1,892 & 1,317 & 1,315 & 1,315 & 1,328 & 1,315 & 3.05 \\
\hline 53 & 1,164 & 791 & 796 & 791 & 781 & 781 & 2.78 \\
\hline 54 & 1,351 & 943 & 940 & 940 & 932 & 932 & 2.83 \\
\hline 55 & 1,404 & 851 & 847 & 847 & 885 & 847 & 3.15 \\
\hline 56 & 2,002 & 1,304 & 1,297 & 1,297 & 1,305 & 1,297 & 3.12 \\
\hline 57 & 1,576 & 1,107 & 1,106 & 1,106 & 1,117 & 1,106 & 4.28 \\
\hline 58 & 2,047 & 1,530 & 1,534 & 1,530 & 1,525 & 1,525 & 4.30 \\
\hline 59 & 2,166 & 1,335 & 1,330 & 1,330 & 1,363 & 1,330 & 4.88 \\
\hline 60 & 2,166 & 1,334 & 1,336 & 1,334 & 1,356 & 1,334 & 4.82 \\
\hline
\end{tabular}


Table 11 Computational results for data set 2 with $R=30$ and $L=20$ (continued)

\begin{tabular}{|c|c|c|c|c|c|c|c|}
\hline \multirow[b]{2}{*}{ No } & \multirow[b]{2}{*}{ Initial cost } & \multicolumn{3}{|c|}{$H A S$} & \multirow[b]{2}{*}{ Simulated annealing } & \multirow[b]{2}{*}{ Best of all } & \multirow[b]{2}{*}{ Time } \\
\hline & & HASI & HASII & Best & & & \\
\hline$\overline{61}$ & 1,677 & 1,134 & 1,138 & 1,134 & 1,154 & 1,134 & 4.43 \\
\hline 62 & 2,416 & 1,697 & 1,693 & 1,693 & 1,706 & 1,693 & 4.38 \\
\hline 63 & 2,494 & 1,638 & 1,632 & 1,632 & 1,681 & 1,632 & 5.03 \\
\hline 64 & 3,775 & 2,411 & 2,409 & 2,409 & 2,441 & 2,409 & 4.97 \\
\hline 65 & 2,265 & 1,544 & 1,539 & 1,539 & 1,553 & 1,539 & 5.90 \\
\hline 66 & 3,094 & 2,224 & 2,224 & 2,224 & 2,242 & 2,224 & 5.90 \\
\hline 67 & 3,025 & 2,074 & 2,075 & 2,074 & 2,107 & 2,074 & 6.80 \\
\hline 68 & 4,364 & 3,031 & 3,025 & 3,025 & 3,051 & 3,025 & 6.70 \\
\hline 69 & 2,728 & 1,708 & 1,706 & 1,706 & 1,720 & 1,706 & 6.22 \\
\hline 70 & 3,646 & 2,472 & 2,469 & 2,469 & 2,525 & 2,469 & 6.20 \\
\hline 71 & 3,531 & 2,425 & 2,436 & 2,425 & 2,447 & 2,425 & 7.18 \\
\hline 72 & 5,223 & 3,547 & 3,547 & 3,547 & 3,642 & 3,547 & 7.15 \\
\hline
\end{tabular}

Table 12 Computational results for data set 2 with $R=48$ and $L=32$

\begin{tabular}{lccccccc}
\hline & & \multicolumn{7}{c}{$H A S$} & & & \\
\cline { 3 - 5 } No & Initial cost & $H A S I$ & HASII & Best & Simulated annealing & Best of all & Time \\
\hline 73 & 2,022 & 1,347 & $\mathbf{1 , 3 4 0}$ & $\mathbf{1 , 3 4 0}$ & 1,373 & 1,340 & 17.28 \\
74 & 2,101 & 1,543 & $\mathbf{1 , 5 4 0}$ & $\mathbf{1 , 5 4 0}$ & 1,548 & 1,540 & 17.23 \\
75 & 2,640 & 1,736 & 1,729 & 1,729 & $\mathbf{1 , 7 0 5}$ & 1,705 & 18.93 \\
76 & 3,636 & 2,560 & 2,556 & 2,556 & $\mathbf{2 , 5 3 8}$ & 2,538 & 18.48 \\
77 & 2,024 & $\mathbf{1 , 2 0 0}$ & $\mathbf{1 , 2 0 0}$ & $\mathbf{1 , 2 0 0}$ & 1,215 & 1,200 & 16.22 \\
78 & 2,436 & $\mathbf{1 , 6 3 4}$ & 1,636 & $\mathbf{1 , 6 3 4}$ & 1,645 & 1,634 & 16.25 \\
79 & 3,001 & 1,710 & $\mathbf{1 , 7 0 6}$ & $\mathbf{1 , 7 0 6}$ & 1,720 & 1,706 & 18.52 \\
80 & 4,323 & 2,496 & 2,493 & 2,493 & $\mathbf{2 , 4 8 3}$ & 2,483 & 18.57 \\
81 & 2,933 & $\mathbf{1 , 9 8 4}$ & $\mathbf{1 , 9 8 4}$ & $\mathbf{1 , 9 8 4}$ & 2,034 & 1,984 & 27.50 \\
82 & 4,445 & 2,936 & $\mathbf{2 , 9 2 7}$ & $\mathbf{2 , 9 2 7}$ & 2,937 & 2,927 & 27.17 \\
83 & 4,924 & 2,910 & $\mathbf{2 , 9 0 7}$ & $\mathbf{2 , 9 0 7}$ & 2,962 & 2,907 & 30.27 \\
84 & 6,585 & 4,008 & $\mathbf{4 , 0 0 5}$ & $\mathbf{4 , 0 0 5}$ & 4,054 & 4,005 & 29.85 \\
85 & 3,241 & $\mathbf{1 , 9 7 6}$ & $\mathbf{1 , 9 7 6}$ & $\mathbf{1 , 9 7 6}$ & 1,978 & 1,976 & 26.78 \\
86 & 4,787 & 3,102 & 3,104 & 3,102 & $\mathbf{3 , 0 8 7}$ & 3,087 & 26.97 \\
87 & 5,082 & 2,914 & $\mathbf{2 , 9 1 0}$ & $\mathbf{2 , 9 1 0}$ & 3,007 & 2,910 & 30.62 \\
88 & 6,896 & 4,451 & $\mathbf{4 , 4 4 0}$ & $\mathbf{4 , 4 4 0}$ & 4,444 & 4,440 & 30.77 \\
89 & 4,387 & 2,911 & $\mathbf{2 , 9 0 1}$ & $\mathbf{2 , 9 0 1}$ & 2,936 & 2,901 & 36.49 \\
90 & 6,202 & $\mathbf{3 , 9 3 7}$ & 3,939 & $\mathbf{3 , 9 3 7}$ & 4,010 & 3,937 & 39.44 \\
91 & 6,883 & 4,450 & $\mathbf{4 , 4 4 8}$ & $\mathbf{4 , 4 4 8}$ & 4,517 & 4,448 & 41.63 \\
92 & 9,285 & 5,903 & 5,912 & 5,903 & $\mathbf{5 , 9 0 2}$ & 5,902 & 44.98 \\
93 & 4,866 & 2,870 & $\mathbf{2 , 8 6 2}$ & $\mathbf{2 , 8 6 2}$ & 2,925 & 2,862 & 40.66 \\
94 & 5,982 & 3,968 & $\mathbf{3 , 9 6 0}$ & $\mathbf{3 , 9 6 0}$ & 4,070 & 3,960 & 41.78 \\
95 & 7,741 & $\mathbf{4 , 7 6 7}$ & $\mathbf{4 , 7 6 7}$ & $\mathbf{4 , 7 6 7}$ & 4,777 & 4,767 & 49.56 \\
96 & 10,542 & 6,523 & $\mathbf{6 , 5 2 1}$ & $\mathbf{6 , 5 2 1}$ & 6,655 & 6,521 & 48.17 \\
\hline & & & & & & &
\end{tabular}




\section{Conclusions}

In this article, a mathematical model and two HASs were presented to solve the CSP. More specifically, a binary integer linear programming model was presented for the CSP. Since the CSP is computationally intractable and only small-size problems can be solved using the mathematical model, two HASs were presented for the CSP. The first HAS (HAS I) is a direct modification of HAS-QAP for the CSP. The second HAS (HAS II) uses a simulated annealing heuristic, instead of a random descent heuristic as in HAS I. The proposed heuristics performed well on two data sets presented in the literature.

\section{Acknowledgements}

The authors express sincere thanks to the anonymous reviewers for their valuable suggestions.

\section{References}

Bouleimen, K. and Lecocq, H. (2003) 'A new efficient simulated annealing algorithm for the resource-constrained project scheduling problem and its multiple mode version', European Journal of Operational Research, Vol. 149, No. 2, pp.268-281.

Charikar, M., Khuller, S. and Raghavachari, B. (2002) 'Algorithms for capacitated vehicle routing', SIAM Journal on Computing, Vol. 31, No. 3, pp.665-682.

Christofides, N. and Colloff, I. (1973) 'The rearrangement of items in a warehouse', Operations Research, Vol. 21, No. 2, pp.577-589.

Colorni, A., Dorigo, M. and Maniezzo, V. (1991) 'Distributed optimization by ants colonies', in F. Varela and P. Bourgine (Eds), First European Conference on Artificial Life, pp.134-142.

Dorigo, M. and Gambardella, L.M. (1997) 'Ant colony system: a cooperative learning approach to the traveling salesman problem', IEEE Transactions on Evolutionary Computation, Vol. 1, No. 1, pp.53-66.

Dorigo, M., Maniezzo, V. and Colorni, A. (1996) 'The ant system: optimization by a colony of cooperating agents', IEEE Transactions on Systems, Man, and Cybernetics - PartB, Vol. 26, No. 1, pp.29-41.

Gambardella, L.M., Taillard, E.D. and Dorigo, M. (1999) 'Ant colonies for the quadratic assignment problem', Journal of Operations Research Society, Vol. 50, No. 2, pp.167-176.

Maniezzo, V. and Colorni, A. (1999) 'The ant system applied to the quadratic assignment problem', IEEE Transactions on Knowledge and Data Engineering, Vol. 11, No. 5, pp.769-778.

McKendall, A.R., Jr. and Shang, J. (2006) 'Hybrid ant systems for the dynamic facility layout problem', Computers and Operations Research, Vol. 33, No. 3, pp.790-803.

McKendall, A.R., Jr. and Shang, J. (2008) 'Tabu search heuristics for the crane sequencing problem', Int. J. Operational Research, Vol. 3, No. 4, pp.412-429.

McKendall, A.R., Jr., Noble, J.S. and Klein, C.M. (2005) 'Simulated annealing heuristics for managing resources during planned outages at electric power plants', Computers and Operations Research, Vol. 32, No. 1, pp.107-125. 
McKendall, A.R., Jr., Shang, J., Noble, J.S. and Klein, C.M. (2006) 'A simulated annealing heuristic for a crane sequencing problem', Int. J. Industrial Engineering, Vol. 13, No. 1, pp.90-98.

Stutzle, T. and Dorigo, M. (1999) 'ACO algorithms for the quadratic assignment problem', in D. Corne, M. Dorigo and F. Glover (Eds), New Ideas in Optimization. New York, NY: McGraw-Hill, pp.33-50. 